\title{
Veränderungen in der Färbbarkeit mit lipoidfärbendem Viktoriablau bei den von Pankreatin angegriffenen Leberzellen.
}

\author{
パンクレアチンが作用した肝細胞の類脂質染料なる \\ ビクトリア青での染色性の変化.
}

\author{
Yoshiki TAMURA 田村良喜.
}

[Eingegangen am 26. November 1954.]

In meinem vorigen Versuche (1954) habe jch beobachtet, daß in den Leberzellen der Mäuse mit speziell lipoidfärbendem Viktoriablau gut färbbare Granula reichlich vorhanden sind, und daß diese, wenn die Versuchstiere mit Lecithin intravenös injiziert werden, sich noch vermehren. Es wurde von MORI (1954) festgestellt, daß die mit dem Eiweißverdauungsferment Pankreatin versetzten Milchkügelchen in der Kuhmilch im Verlauf der Zeit mit Viktoriablau immer stärker und in größerer Zahl färbbar werden, daß ferner bei der Fütterung der Mäuse mit der Kuhmilch die in den Magen und Darm gelangten Milchkügelchen mit Viktoriablau sehr stark färbbar werden. In der vorliegenden Arbeit ließ ich das Pankreatin auf die Leberschnitte der Maus einwirken und beobachtete die Zellen nach der Färbung mit Viktoriablau.

\section{Material und Methode.}

Die Mäuseleber wurde herausgenommen, in eine physiologische Kochsalzlösung gebracht, und aus ihr wurden $40 \mu$ dicke Gefrierschnitte hergestellt. Man übertrug die Schnitte aus der physiologischen Kochsalzlösung in eine mit derselben Lösung bereitete auf $25^{\circ} \mathrm{C}$ gehaltene 0.01\% ige Pankreatinlösung von pH7.5. Nach 5, 10 und 20 Minuten wurden die Schnitte für 3 Minuten in eine mit physiologischer Kochsalzlösung bereitete 1\%ige Formalinlösung gebracht, um die Wirkung des Pankreatins umzusetzen. Nach etwa 3minutiger Ausspülung im Wasser wurden die Schnitte schließlich in eine 0.01\% ige Viktoriablaulösung eingelegt, die ebenfalls mit physiologischer Kochsalzlösung bereitet wurde. Man bereitete diese Farbstofflösung, richtiger Farbstoffsuspension, indem man eine $1 \%$ ige alkoholische Viktoriablaulösung mit der Kochsalzlösung $300 \mathrm{fach}$ verdünnte. Die in ihr 10 Minuten gefärbten Schnitte wurden kurz abgespült, auf ein Objektglas gebracht, abgetropft, vor dem Ab- 
trocknen im Gummisirup, bestehend aus Gummi arabicum 5, Rohrzucker 5 und Wasser 20, eingeschlossen und mikroskopiert. In diesem wässerigen Einschlußmedium werden die Zellen allerdings nicht hinreichend aufgehellt. Die feinsten Gebilde in ihnen waren also nicht sehr deutlich zu sehen, aber das Ziel meiner Untersuchung konnte im Großen erreicht werden. Kontrollversuche an den nicht mit Pankreatin versetzten Gefrierschnitten liefen parallel.

\section{Ergebnisse der Untersuchung.}

\section{a) Färbung der unbehandelten Leberschnitte.}

Wie man aus Abb. 1 ersieht, finden sich blau färbbare Granula verschiedener Größe im ganzen Bereich des Zelleibes der Leberzellen.

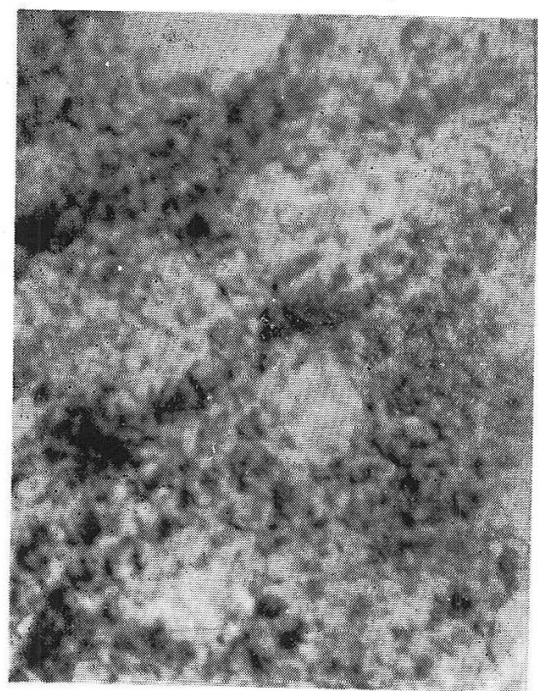

Abb. 1. Viktoriablaufärbung des nicht fixierten Gefrierschnittes der Mäuseleber. Die feinste Struktur ist infolge des den Schnitt umgebenden wässerigen Einschlußmittels nicht sehr deutlich, man sieht aber außer den vielen rundlichen und polymorphen Körperchen von verschiedener Größe und Färbungsstärke noch zahlreiche als Mitochondrien anzusehende kleine stäbchenförmige, ovale und runde Gebilde. $960 \times$.
Ein gleiches Bild habe ich früher (1953) an zuvor mit Formalin fixierten Leberschnitten beobachtet. Unter den mit Viktoriablau gefärbten Granula gibt es fadenförmige, stäbchenförmige, rundliche und ovale. Die fadenund stäbchenförmigen sind wahrscheinlich Mitochondrien und finden sich in der Nähe der Kernes verhältnismäßig zahlreich. Die runden und ovalen sind ohne Zweifel zum Teil Mitochondrien und zum größten 'Teil aus Eiweiß bestehende Einschlüße und fettige Körperchen. Außerdem sieht man $\mathrm{zwischen}$ solchen gefärbten Granula zahl. reich unregelmäßig gestaltete, nicht gut färbbare Körperchen. Die kleinen lipoiden Einschlüße in den mit $\mathrm{OsO}_{4}$ fixierten Leberzellen, die von PALADE (195̄2) im Elektronenmikroskop beobachtet wurden, färben sich mit Viktoriablau ziemlich stark.

Die Cytoplasmagrundsubstanz zwischen den gefärbten Körperchen wird schwach gefärbt. Das zeigt, daß in ihr Lipoide, wenn auch gering, enthalten sind. 


\section{b) Färbung nach 5 Minuten langer Wirkung des Pankreatins.}

Die mit Viktoriablau stark färbbaren Granula von verschiedener Größe nehmen an Zahl und an Färbestärke bedeutend zu (Abb. 2). Die besonders stark dunkel färbbaren Granula sind verhältnismäßig groß und verteilen sich im ganzen Bereich der Zellen. Auch die Färbbarkeit der kleinsten Granula und der Mitochondrien verstärkt sich, wenn auch nur in geringem Maßc. BENSLEY (1937) trennte die Mitochondrien aus der Emulsion der Leberzellen durch Centrifugierung $a b$ und untersuchte sie chemisch. Nach ihm sollen die Bestandteile der Mitochondrien folgende sein : Proteine und Unbekannte $64.67 \%$, Glyceride $28.88 \%$, Lecithin, Cephalin etc. $42 \%$ und Cholesterol 2.25\%. Es ist leichtverständlich, daß infolge der Wirkung des Pankreatins die Eiweißsubstanz der Mitochondrien zersezt wird, so daß die demaskierten, frei gewordenen Lipoide nun mit Viktoriablau färbbar

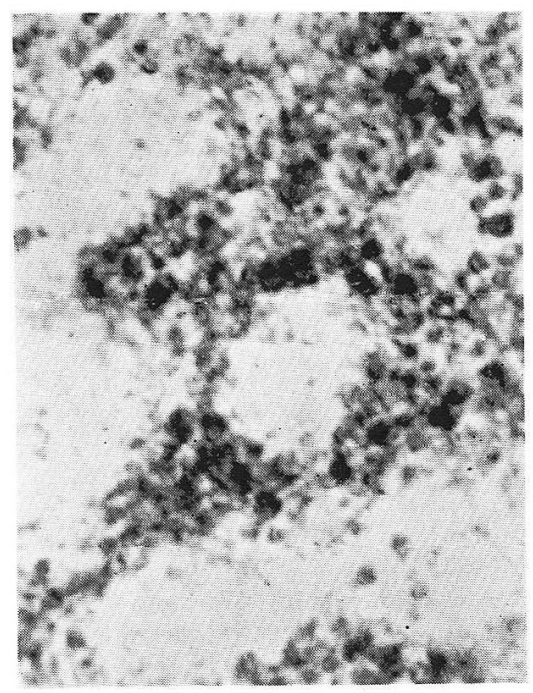

Abb. 2. Viktoriablaufärbung des Schnittes nach 5 minutiger Wirkung des Pankreatins. Die Färbbarkeit der Körperchen in den Leberzellen, einschließlich der Mitochondrien, verstärkt sich. $960 \times$. werden.

Die Färbung der Cytoplasmagrundsubstanz steigert sich nach der Wirkung des Pankreatins nicht, weil die Grundsubstanz so zart und substanzarm ist, daß durch die Wirkung des Pankreatins die demaskierten Lipoide mit den zersetzten Eiweißsubstanzen in kurzer Zeit fast restlos verloren gehen.

\section{c) Färbung nach 10 Minuten langer Wirkung des Pankreatins.}

Die Färbbarkeit der verschiedenen Granula in den Leberzellen mit Viktoriablau steigert sich noch mehr, und die Unterscheidung der Mitochondrien von den anderen Granula ist fast unmöglich (Abb. 3). Die Cytoplasmagrundsubstanz ist kaum zu bemerken.

d) Färbung nach 20 Minuten langer Wirkung des Pankreatins.

Die meisten Gebilde in den Leberzellen färben sich slark, und der Zelleib erscheint mit den so tingierten Gebilden vollpfropft (Abb. 4). 


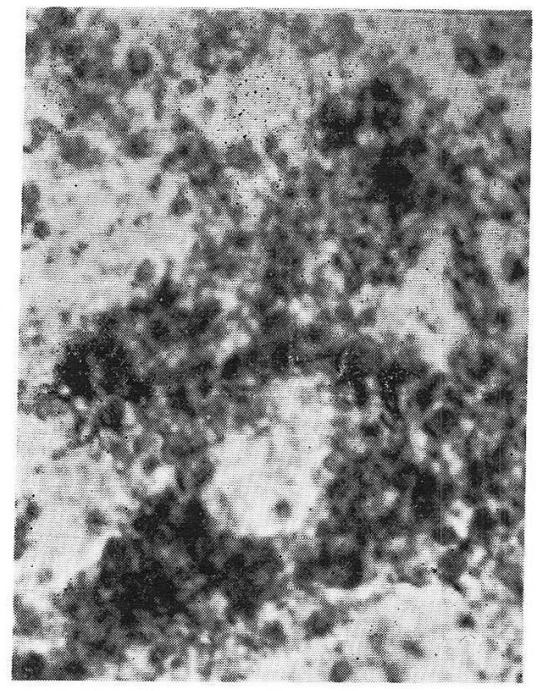

Abb. 3. Viktoriablaufärbung des Schnittes nach 10minutiger Wirkung des Pankreatins. Die Färbungstärke der Gebilde in den Leberzellen steigert sich noch mehr. Die Cytoplasmagrundsubstanz ist größtenteils ausgelöst. $960 \times$.

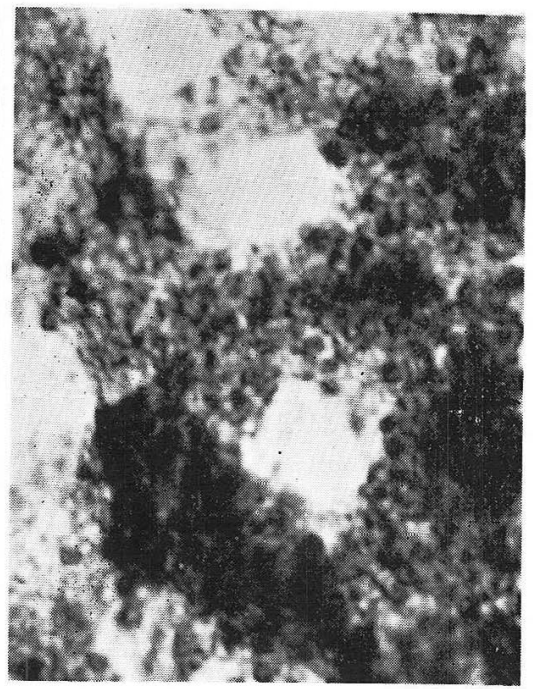

Abb. 4. Vik toriablaufärbung des Schnittes nach 20minutiger Wirkung des Pankreatins. Alle Gebilde in den Leberzellen färben sich sehr stark. 960x.

\section{Zusammenfassung.}

Man ließ das Pankreatin auf die Gefrierschnitte der frischen Mäuseleber einwirken und stellte fest, daß die in den Leberzellen vorhandenen Mitochondrien und andere Körpcrchen (verschiedene Zelleinschlüße, fettige Körperchen usw.) mit dem Viktoriablau stark färbbar werden. Dies beruht offenbar auf der Lemaskierung der Lipoide in diesen Gebilden.

\section{内 容自 抄.}

二十月鼠の生の肺の凍結比版パンクレアチンを作用させ，ビクトリア 青で染め, 朋細胞内の䊀子の染色の状洸を観察した。パンクレアチンとよ

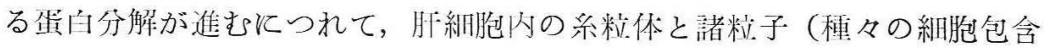
物，脂肪性:の粘子等）がビクトリア青に独く染まるようになる。これはそ れ筞の物象の颣脂質が航仪面されるためと解せられる。

\section{Literatur.}

Bensley, R. R.: On the fat distribution in mitochondria of the guinea 
pig liver. Anat. Rec. 69 (1937). - Mori, Ch. : Morphologische Studien über die Verdauung der Milchkügelchen. Arch. hist. jap. 6 (1954). - Palade, G. E. : A study of fixation for electron microscopy. J. exp. Med. 95 (1952).

Tamura, Y.: Viktoriablau-Färbung der Schnitte von Leber, Niere und Lymphknoten der mit Lecithin intravenös injizierten Mäuse. Arch. hist. jap. 6 (1954). 